\title{
Exploring the influence of transfer channels on fusion reactions: the case of ${ }^{40} \mathrm{Ca}+{ }^{58,64} \mathrm{Ni}$
}

\author{
D. Bourgin ${ }^{1}$, S. Courtin ${ }^{1}$,a, F. Haas ${ }^{1}$, A. Goasduff ${ }^{1}$, A.M. Stefanini ${ }^{2}$, G. Montagnoli ${ }^{3}$, D. Montanari ${ }^{1}$, L. Corradi ${ }^{2}$, J. \\ Huiming $^{2}$, F. Scarlassara ${ }^{3}$, E. Fioretto ${ }^{2}$, C. Simenel ${ }^{4}$, N. Rowley ${ }^{5}$, C.L. Jiang ${ }^{6}$, S. Szilner ${ }^{7}$, and T. Mijatović ${ }^{7}$ \\ ${ }^{1}$ Institut Pluridisciplinaire Hubert Curien UMR7178 IN2P3-CNRS, 23 rue du Loess F-67037 Strasbourg \\ ${ }^{2}$ INFN, Laboratori Nazionali di Legnaro, I-35020 Legnaro (Padova), Italy \\ ${ }^{3}$ Dipartimento di Fisica, Universita di Padova, and INFN, Sezione di Padova, I-35131 Padova, Italy \\ ${ }^{4}$ Department of Nuclear Physics, RSPE, Australian National University, Canberra, Australian Capital Territory 2601, Australia \\ ${ }^{5}$ Institut de Physique Nucléaire, Orsay, France \\ ${ }^{6}$ Argonne National Laboratory, Argonne, USA \\ ${ }^{7}$ Rudler Bosskovic Institute, HR-10002 Zagreb, Croatia
}

\begin{abstract}
Fusion cross sections have been measured in the ${ }^{40} \mathrm{Ca}+{ }^{58} \mathrm{Ni}$ and ${ }^{40} \mathrm{Ca}+{ }^{64} \mathrm{Ni}$ systems at beam energies ranging from $\mathrm{E}_{\text {lab }}=104.75 \mathrm{MeV}$ to $153.5 \mathrm{MeV}$ using the Laboratori Nazionali di Legnaro electrostatic deflector. Distributions of barriers have been extracted from the experimental data. Preliminary coupled channel calculations were performed and hints of effects of neutron transfers on the fusion below the barrier in the ${ }^{40} \mathrm{Ca}$ $+{ }^{64} \mathrm{Ni}$ are discussed.
\end{abstract}

\section{Introduction}

Fusion-evaporation is the dominant reaction mechanism in medium-light heavy-ion collisions at relatively low bombarding energies. This contribution discusses fusion at and below the Coulomb barrier (CB) energies for the two ${ }^{40} \mathrm{Ca}$ $+{ }^{58} \mathrm{Ni}$ and ${ }^{40} \mathrm{Ca}+{ }^{64} \mathrm{Ni}$ systems. It has been demonstrated that the dominant features observed at these energies, are the enhancement of the fusion cross-section at the $\mathrm{CB}$ and at moderate subbarrier energies, and the hindrance of the cross-section at deep subbarrier energies. Fusion crosssections around the $\mathrm{CB}$ are indeed driven by couplings of the relative motion of the colliding nuclei to their low energy surface vibrations and/or stable deformations. These couplings can be included in coupled-channels (CC) calculations to calculate fusion features around the $\mathrm{CB}$. The $\mathrm{CC}$ calculations of the distributions of barriers $(\mathrm{D}(\mathrm{B}))$ and their extraction from precise cross-section measurements have revealed to be a powerful tool to better understand the role of couplings to collective degrees of freedom of the target and projectile.

At much lower energies, the measured cross-sections have been systematically found to decrease sharply, well below predictions of usual CC calculations based on standard Woods-Saxon potentials. Contrary to the fusion enhancement near the $\mathrm{CB}$, the fusion hindrance phenomenon at lower energies has only been recently observed in a large number of collisions and its strength depends strongly on the nuclear structure of the interacting nuclei.

\section{Previous results concerning the $\mathrm{Ca}+\mathrm{Ca}$ and $\mathrm{Ni}+\mathrm{Ni}$ systems}

We recently performed several studies on the fusion hindrance, especially in medium mass systems. In the context of this paper, we would first like to review briefly the results obtained for the the $\mathrm{Ca}+\mathrm{Ca}$ and $\mathrm{Ni}+\mathrm{Ni}$ systems where fusion cross sections have been measured from well above the barrier down to very low cross section[1, 2, 4, 5]. All systems studied, except ${ }^{40} \mathrm{Ca}+{ }^{48} \mathrm{Ca}$ have negative $\mathrm{Q}$ values for fusion.

What the $\mathrm{Ca}+\mathrm{Ca}$ reactions are concerned, it has been observed that:

- All systems show large fusion hindrance at deep subbarrier energies, but the effect is different for ${ }^{40} \mathrm{Ca}+{ }^{40} \mathrm{Ca}$ and ${ }^{40} \mathrm{Ca}+{ }^{48} \mathrm{Ca}$. This is probably due to the influence of the strong $3^{-}$and of the $5^{-}$states in the soft ${ }^{40} \mathrm{Ca}$ nucleus (even if it is a magic nucleus) in comparison with the rather stiff ${ }^{48}$ Ca nucleus.

- In the asymmetric ${ }^{40} \mathrm{Ca}+{ }^{48} \mathrm{Ca}$ system, the hindrance effect shows up at lower energies and the excitation function crosses the ${ }^{48} \mathrm{Ca}+{ }^{48} \mathrm{Ca}$ one around $\mathrm{E}_{\mathrm{CM}} \simeq 50 \mathrm{MeV}$. This can be seen in Fig. 1, showing our latest cross section results for ${ }^{40,48} \mathrm{Ca}+{ }^{40,48} \mathrm{Ca}$ [1]. All structure characteristics of the target and projectile have been taken into account and we have shown that it is necessary to include transfer channels effects to reproduce the ${ }^{40} \mathrm{Ca}+{ }^{48} \mathrm{Ca}$ data. Interestingly enough, $Q$ values for the $+2 n,+3 n$ and $+4 n$ transfer channels (from ${ }^{48} \mathrm{Ca}$ towards ${ }^{40} \mathrm{Ca}$ ) are respectively $+2.62,+0.16$ and $+3.87 \mathrm{MeV}$ and $\mathrm{Q}$ values for the $-1 \mathrm{p},-2 \mathrm{p},-3 \mathrm{p},-4 \mathrm{p}$ and $-5 \mathrm{p}$ are $+1.30,+7.08,+4.90,+7.02$

\footnotetext{
ae-mail: sandrine.courtin@iphc.cnrs.fr
} 
and $+0.48 \mathrm{MeV}$ respectively, all other transfer channels having negative $Q$ values, as it is also the case for ${ }^{40} \mathrm{Ca}+$ ${ }^{40} \mathrm{Ca}$ and ${ }^{48} \mathrm{Ca}+{ }^{48} \mathrm{Ca}$.

\section{The experimental study of the fusion of ${ }^{40} \mathrm{Ca}+{ }^{58,64} \mathrm{Ni}$}

Table 1. Corrected $\mathrm{Q}$ values for transfer reactions for the systems 1) ${ }^{40} \mathrm{Ca}+{ }^{58} \mathrm{Ni}$ and 2) ${ }^{40} \mathrm{Ca}+{ }^{58} \mathrm{Ni}$

$\left(Q_{\text {corr }}=Q_{\text {transfer }}+V_{b, \text { in }}^{C}-V_{b, \text { out }}^{C}\right)$. The indicated + sign corresponds to (n or $\mathrm{p}$ ) transfers from target $(\mathrm{Ni})$ to projectile (Ca) and the - sign to transfers from projectile $(\mathrm{Ca})$ to target (Ni). The non indicated other transfer channels have larger negative $Q$ values.

\begin{tabular}{ccccc}
\hline System & $+1 \mathrm{n}$ & $+2 \mathrm{n}$ & $+3 \mathrm{n}$ & $+4 \mathrm{n}$ \\
\hline 1 & -3.80 & -2.52 & -11.19 & -14.21 \\
2 & -1.23 & 3.47 & 0.86 & 4.22 \\
\hline & & & & \\
\hline System & $-1 \mathrm{p}$ & $-2 \mathrm{p}$ & $-3 \mathrm{p}$ & $-4 \mathrm{p}$ \\
\hline 1 & -3.75 & -3.60 & -11.95 & -15.97 \\
2 & 0.26 & 4.19 & 0.88 & 1.81 \\
\hline
\end{tabular}

What the $\mathrm{Ca}+\mathrm{Ni}$ systems are concerned, in previous pionneering work by Sikora et al. [9], more than 30 years ago, fusion excitation functions for ${ }^{40} \mathrm{Ca}+{ }^{58} \mathrm{Ni}$ have been measured at energies above the barrier ranging from $\mathrm{E}_{\mathrm{lab}}$ $=113$ to $170 \mathrm{MeV}$ to extract information on the potentials and eventual neutron effecs. To our knowledge, this is the only published fusion excitation function result for the $\mathrm{Ca}+\mathrm{Ni}$ systems. The authors have also measured other $\mathrm{Ca}+\mathrm{Ni}$ systems but with very limited number of energy points.

We are particularly interested in the influence of transfer channels on the fusion cross sections. This study has thus been performed at bombarding energies where the fusion hindrance is still not very effective. Table 1 presents the corrected Q values for transfer channels in the ${ }^{40} \mathrm{Ca}+$ $58,64 \mathrm{Ni}$ reactions.

Interestingly enough these $\mathrm{Q}$ values are positive for $+2 \mathrm{n}$ to $+4 \mathrm{n}$, and for $-1 \mathrm{p}$ to $-4 \mathrm{p}$ for ${ }^{40} \mathrm{Ca}+{ }^{64} \mathrm{Ni}$ and negative for all transfer channels for the ${ }^{40} \mathrm{Ca}+{ }^{58} \mathrm{Ni}$ system. The structure of the colliding nuclei is very known. As will be discussed later, ${ }^{58} \mathrm{Ni}$ and ${ }^{64} \mathrm{Ni}$ have very similar structure and the main difference between these systems is the different number of neutrons $(+6 n)$ in the case of ${ }^{40} \mathrm{Ca}+{ }^{64} \mathrm{Ni}$. In this study, we have taken advantage of the unique intense LNL XTU Tandem ${ }^{40} \mathrm{Ca}$ negative-ion beam and of the LNL electrostatic deflector to measure accurately the cross sections of the fusion reactions ${ }^{40} \mathrm{Ca}$ $+{ }^{58,64} \mathrm{Ni}$. The experiment has been performed at beam energies ranging from $\mathrm{E}_{l a b}=104.75 \mathrm{MeV}$ to $153.5 \mathrm{Mev}$ in steps of $1.25 \mathrm{MeV}$ below the Coulomb barrier and 2.5 $\mathrm{MeV}$ above the Coulomb barrier. The ${ }^{40} \mathrm{Ca}$ beam was impinging on $50 \mu \mathrm{g} . \mathrm{cm}-2{ }^{58} \mathrm{Ni}$ and ${ }^{64} \mathrm{Ni}$ targets deposited on a $20 \mu \mathrm{g} . \mathrm{cm}-2{ }^{12} \mathrm{C}$ backing. The recoiling evaporation residues (ER) were separated from the incident beam at forward angles using the LNL electrostatic deflector at $2^{\circ}$
[12]. This allowed us to measure a very accurate excitation function for both systems and to extract distributions of barriers from above the $\mathrm{CB}$ to lower energies where the cross section is still not affected by fusion hindrance features.

\section{Preliminary results}

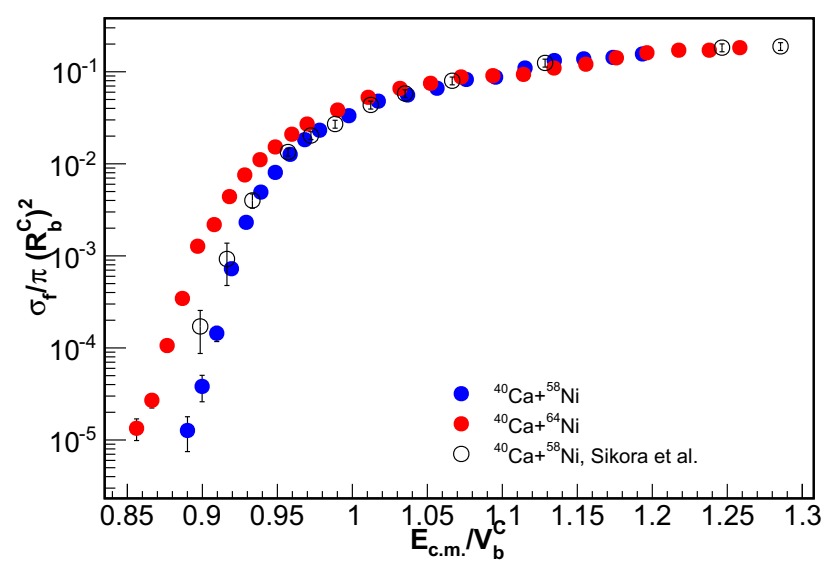

Figure 1. Reduced experimental fusion excitation functions for the ${ }^{40} \mathrm{Ca}+{ }^{58,64} \mathrm{Ni}$ systems measured in this study. Blue circles are for the ${ }^{40} \mathrm{Ca}+{ }^{58} \mathrm{Ni}$ system and red circles are for the ${ }^{40} \mathrm{Ca}$ $+{ }^{64} \mathrm{Ni}$. Previous results for the ${ }^{40} \mathrm{Ca}+{ }^{58} \mathrm{Ni}$ system are reported with white circles. Preliminary $\mathrm{CC}$ calculations are also reported (see text).

Figure 1 shows the measured reduced excitations functions for the ${ }^{40} \mathrm{Ca}+{ }^{58,64} \mathrm{Ni}$ systems. The previous Sikora results for the ${ }^{40} \mathrm{Ca}+{ }^{58} \mathrm{Ni}$ [9] system are also reported, in good agreement for the present measurement, except the last Sikora point, which should be noted is affected by 50 $\%$ error bar. The trend at low energies for the reduced ${ }^{40} \mathrm{Ca}$ $+{ }^{64} \mathrm{Ni}$ cross-section shows a different behavior, which is a first indication that some physics may be different for this system.

Preliminary coupled channel (CC) calculations have been performed using the CCFULL [10] code and are also reported on this Figure. As a first step main structure features of the colliding nuclei have been included in the calculation. These are COUPLINGS; The potential used is the global Akyüz and Winther (AW) potential [11] with standard geometry $\mathrm{r}_{0}=1.18 \mathrm{fm}$ and $\mathrm{a}=0.66$. This leads to fusion barrier positions and heights $\mathrm{R}_{B}^{A W}=10.15$ and $10.36 \mathrm{fm}$ and $\mathrm{V}_{B}^{A W}=73.81$ and $72.47 \mathrm{MeV}$ for ${ }^{40} \mathrm{Ca}+{ }^{58} \mathrm{Ni}$ and ${ }^{40} \mathrm{Ca}+{ }^{64} \mathrm{Ni}$ respectively. These calculations are in good agreement with the experimental data for the ${ }^{40} \mathrm{Ca}+$ ${ }^{58} \mathrm{Ni}$ system at the barrier, and the calculation with the bare AW potential gives a good description of the data above CB.

\section{Acknowledgments}

We would like to thank the professional work of the XTU Tandem and target LNL laboratory staff. The research 
leading to these results has received funding from the European Union Seventh Framework Programme under Grant Agreement No. 262010 ENSAR. A. G. was supported by the P2IO Excellence Laboratory.

\section{References}

[1] G. Montagnoli et al. Phys.Rev. C 85, 024607 (2012) and references therein.

[2] M. Beckerman et al., Phys.Rev. C 231581 (1981).

[3] M. Beckerman et al., Phys.Rev.Lett. C 451472 (1980).

[4] A.M. Stefanini et al., Phys. Rev. Lett. 74864 (1995).

[5] C.L. Jiang et al., Nucl.Phys. A834, 615c (2010) and references therein.
[6] A.M. Stefanini et al., Phys. Rev. C 81037601 (2010).

[7] H. Timmers et al., Nucl.Phys. A 633421 (1998).

[8] A.M. Stefanini et al., Phys. Rev. C 76014610 (2007).

[9] B. Sikora et al., Phys.Rev. C 202219 (1979).

[10] K. Hagino et al., Comput. Phys. Commun. C 123143 (1999).

[11] O. Akyüz, A. Winther, in Nuclear Structure and Heavy-Ion Physics, Proc. Int. School of Physics, Enrico Fermi, Varenna, North-Holland, Amsterdam (1981) 492.

[12] S. Beghini et al., Nucl. Instrum. and Methods A 239 585 (1985).

[13] A.M. Stefanini et al., Phys. Rev. C 82014614 (2010). 